\title{
Second Critical Point in First Order Metal-Insulator Transitions
}

\author{
Ivan Z. Kostadinov and Bruce R. Patton* \\ Department of Physics, The Ohio State University, Columbus, Ohio 43210, USA
}

(Received 23 June 2008; published 26 November 2008)

\begin{abstract}
For first order metal-insulator transitions we show that, together with the dc conductance zero, there is a second critical point where the dielectric constant becomes zero and further turns negative. At this point the metallic reflectivity sharply increases. The two points can be separated by a phase separation state in a $3 \mathrm{D}$ disordered system but may tend to merge in $2 \mathrm{D}$. For illustration we evaluate the dielectric function in a simple effective medium approximation and show that at the second point it turns negative. We reproduce the experimental data on a typical Mott insulator such as $\mathrm{MnO}$, demonstrating the presence of the two points clearly. We discuss other experiments for studies of the phase separation state and a similar phase separation in superconductors with insulating inclusions.
\end{abstract}

DOI: 10.1103/PhysRevLett.101.226407

The charge transport and metal-insulator transitions (MITs) in disordered systems have been discussed in many papers [1-3], reviews [4,5], and books [6-9]. The initial discussion resulted in agreement that at $T=0$ the transition is completely described by the dc conduction as a function of the Fermi level at $T=0$. This is true for second order MITs, where simultaneously with the vanishing of the conductance the real part of the dielectric function also vanishes. It is negative in the metallic state and positive in the dielectric one, and there is no phase separation in this case. Here we show that in a first order MIT in composites the dc conduction is not enough and the dc dielectric constant has to be considered in order to make the description complete. We will show that in a first order MIT in the composites, in disordered thermodynamically metastable 3D systems, there can be an intermediate two phase region separating the metallic state from the dielectric one, both at $T=0$ and at finite temperatures. We will consider such a metal-dielectric composite with a metallic volume fraction $f$, but our results are relevant for other first order MITs in homogeneous systems, due to the nucleation of metallic inclusions in the dielectric matrix under pressure, for example. In a composite, grains of both components are always present for any metallic volume fraction $f \neq 0$ and $f \neq 1$, and the resistance drop occurs at a volume fraction $f_{c}$ different from the dielectric function sign change $f_{d}$ and in different samples made with different metallic volume fractions. Under pressure due to different compressibility, a composite irreversibly can undergo both transitions. To illustrate this, we show a noncomposite example of the phase separation in the Mott insulator $\mathrm{MnO}$ [10-12]. It demonstrates both the resistance drop near $90 \mathrm{GPa}$ and the subsequent reflectance sharp increase, which becomes pressure-independent at $127 \mathrm{GPa}$ [13]. The data from Ref. [12] are given in Fig. 1, and a detailed discussion of MITs in $\mathrm{MnO}$ is given in [14].

Specific phase separation state.-A metallic 3D sample is screening any static electric fields as well as the electron-
PACS numbers: 71.30.+h, 71.55.Jv, 74.25.Dw, 78.67.-n

electron interaction. Let $f_{c}$ be the metallic volume fraction threshold for the first nonzero conductance to appear. From the percolation theory is known that often the values of $f_{c}$ for 3D systems are smaller than 0.5 and are different for various models [8]. We will prove now for 3D composites that in a first order MIT in the range of values $f>f_{c}$ the sample can be conducting but not screening the external static electric fields. Just above $f_{c}$ the sample is in a larger part made of dielectric grains, which form an infinite cluster of a larger volume than the volume of the metallic infinite cluster, provided that $f_{c}<0.5$. The two coexisting

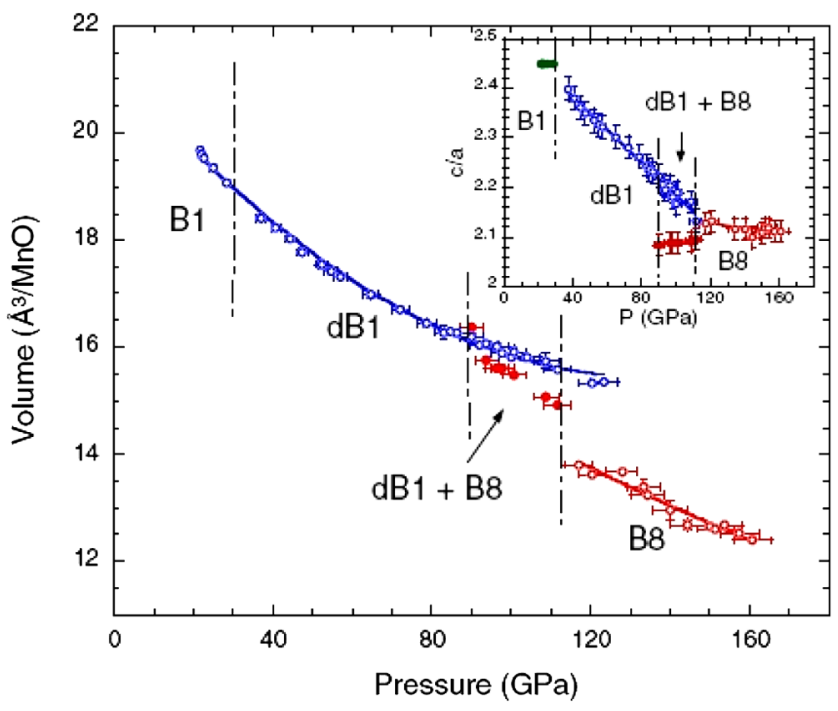

FIG. 1 (color online). From Ref. [12]. The specific volume and the $c / a$ ratio (inset) of $\mathrm{MnO}$ phases (B1, rocksalt; $\mathrm{dB} 1$, a rhombohedral distortion of $\mathrm{B} 1$; and $\mathrm{B} 8$ for NiAs) as a function of pressure. Note the discontinuous changes of the specific volume and the $c / a$ ratio at $110 \mathrm{GPa}$, indicating that $\mathrm{MnO}$ undergoes an isostructural phase transition with $6.6 \%$ volume collapse. This transition coincides within experimental uncertainties with the moment loss and the insulator-metal transition in [11]. 
clusters represent a specific type of phase separation state. Because of the topological structure of the infinite clusters in a disordered system, such phase separation is clearly different from a layered one, for example. When placed in the external static electric field of a capacitor, such a sample, being in a large part dielectric, becomes polarized with nonzero volume polarization. This volume polarization requires energy for creating the positive energy density in the polarized infinite dielectric cluster, which is equal to $\frac{\varepsilon E^{2}}{8 \pi}$, where $\varepsilon$ is the real part of the static (zero frequency) dielectric constant of the sample. The real part of $\varepsilon$ is certainly positive due to the work done for polarizing the sample and not negative as it is in a metal. Thus, we have demonstrated that the phase with both conductance and volume polarization is not a metal nor a dielectric and represents a specific phase separation state. The data presented in Fig. 1 show a separation state as a result of a first order MIT in a different system- $\mathrm{MnO}$, which is not a composite and the metallic phase appears gradually due to nucleation. The resistance drop is near $94 \mathrm{GPa}$ (phases $\mathrm{dB} 1+\mathrm{B} 8$ in Fig. 1, B1 for rocksalt, dB1 for a rhombohedral distortion of $\mathrm{B} 1$, and $\mathrm{B} 8$ for NiAs). The further increase of the metallic volume fraction $f$ breaks the last links between the large dielectric clusters, and all dielectric inclusions become eventually disconnected near $127 \mathrm{GPa}$. Near this point the sample becomes a metallic bulk with isolated dielectric inclusions. The bulk metal has a negative dielectric constant, and at this point (the disappearance of the infinite dielectric cluster) the static dielectric constant turns from positive to negative. The reflectance is pressure-independent above $127 \mathrm{GPa}$ in the bulk metal region. Because of the tunneling of the electrons (holes) and the hybridization of the wave functions of the large dielectric clusters, the real critical point is at values different from the geometric dielectric percolation threshold. To illustrate this second critical point, we applied here a simple version of the effective medium approximation (EMA) to evaluate the dielectric function. Let us mention that the EMA is limited in precision and is one among many similar ones differing in the details $[9,15]$.

Experimental characterization.-Experimentally, one can expect in a first order MIT the metallic reflectance to decrease sharply below this second critical point $f_{d}$, which is different from the sharp resistance drop point $f_{c}$. This is the case with the observed metallic luster in the successive measurements of the same material $\mathrm{MnO}$ [10-12]. Therefore, below the critical point $f_{d}$ the reflectivity decreases sharply due to the transmission of the infinite dielectric cluster and specific plasmons passing through the connected system of tunnels in the metal. The dc polarization $P$ should appear below the point $f_{d}$ in a static electric field in a capacitor, and experiments on its critical behavior are of considerable interest, as are experiments on the behavior of the ac current dissipation in the region, where infinite clusters emerge or disappear. The small dielectric inclusions in the metal will reduce eddy current dissipation at small frequencies, like in an external timedependent magnetic field. When the metal concentration decreases and becomes $f \leq f_{d}$, this eddy current dissipation would have a feature due to the change in the topology of the dielectric inclusions and the appearance of the infinite dielectric cluster, which also marks the bulk metallic volume reduction. Thus the eddy current signal of a coil will show a variation near the second critical point. Another experiment can be carried out in the phase separation state, where one may encounter different sound velocities related to the two infinite clusters with a different type of bonding [16]. In general, we have shown that the dielectric function has a second critical point at $f_{d}$, where the real part of the static $\epsilon$ becomes negative entering the metallic state region. When this critical point does not coincide with the vanishing dc conduction, there will be an intermediate two phase region. In a quasi-2D system made out of parallel infinite metallic cylinders, such phase separation does not exist at all. In a first order MIT, the phase separation region was observed in various Mott insulators like in the $\mathrm{MnO}$ room temperature MIT transition shown here. The experimental data of reference [12] reproduced in Fig. 1 present a volume range in the pressure-volume diagram separating the metallic and the insulating phases of $\mathrm{MnO}$ and showing $6.6 \%$ volume difference. It corresponds to the difference in the metallic volume fraction between the two critical points mentioned above for the case of the composites.

Two types of extended states. - What describes the phase separation state in the range of concentrations $f_{c} \leq f \leq$ $f_{d}$ ? The metallic phase is well defined in the metallic side $f_{d} \leq f \leq 1$, and the insulating phase is as well defined (see below) in the small metallic fraction region $0 \leq f \leq$ $f_{c}$. In the intermediate range $f_{c} \leq f \leq f_{d}$, one has coexistence of the two phases in the form of two infinite clusters. Remarkably, in this region there is also coexistence of two different types of extended states-metallic ones threading the infinite metallic cluster and dielectric ones based on the infinite dielectric cluster. In the dielectric type of extended states between the top and bottom mobility edges, an electron (hole), when injected in the dielectric cluster, can cross the sample as well as it can in the metallic one. For $f \geq f_{d}$ in the metallic phase, only the metallic type of extended states exists, and the sample conductivity is metallic. For $f \leq f_{c}$ in the dielectric phase, only the dielectric type of extended states is present, and, due to the gap, the sample is an insulator at $T=0$. In the vicinity of $f_{c}$ at $T=0$, metallic component states go from localized to delocalized following scaling laws as described by the localization theory $[1,2,5]$. In Table I, the static order parameter values are displayed for all states. The symbol $\mathbf{P}$ is the isotropic dc polarization value.

The second critical point.- In this section we show how the second critical point appears. The two infinite clusters 
TABLE I. The order parameters of metal, insulator, and phase separation states.

\begin{tabular}{lcr}
\hline \hline Insulator & Phase separation state & Metal \\
\hline$\sigma_{\mathrm{dc}}=0$ & $\sigma_{\mathrm{dc}} \neq 0$ & $\sigma_{\mathrm{dc}} \neq 0$ \\
$P \neq 0$ & $P \neq 0$ & $P=0$ \\
\hline \hline
\end{tabular}

and the electronic states based on metallic or dielectric grains and clusters of grains are determined quantum mechanically and not just geometrically. The description also involves the polarization properties of both the metal and the insulator $-\Pi(\omega, f)$. Eventually, the exact form of the metal fraction $f$ dependence of the conductance and the dielectric function and the values of the critical points can vary depending on the models for the calculation. We will use the Drude-type form of $\Pi(\omega, f)$, which is appropriate for illustration purposes. The EMA describes both critical points for the conductance and for the dielectric function, and this is enough to demonstrate how the two critical points appear. A simple version of the EMA (e.g., not accounting for various shapes or coatings of the grains) for the conductivity $\sigma_{\text {eff }}$ of the composite has the form [9]

$$
\Sigma f_{i} \frac{\sigma_{\text {eff }}-\sigma_{i}}{2 \sigma_{\text {eff }}+\sigma_{i}}=0
$$

When the first component $i=1$ is a metal and the second component is an insulator, this equation gives us the first critical point $f_{c}=1 / 3$, which is usually assumed to be the point where the metal-insulator transition happens. Next we find for the same system the static dielectric constant using the same equation as above [9], but with $\varepsilon_{\text {eff }}$ instead of $\sigma_{\text {eff }}$ and $\varepsilon_{1}$ for the metal, the dielectric being marked $i=2$. We neglect here all spectral behavior of the dielectric (and the metal) by assuming a constant $\varepsilon_{\text {diel }}$. For an ideal metal the static dielectric constant is infinite and negative. The above equation gives us $\varepsilon_{\text {eff }}$ of an ideal metal in a dielectric in the form

$$
\varepsilon_{\text {eff }}=\frac{\epsilon_{\text {diel }}}{1-3 f} .
$$

Thus, the ideal metal in a composite with any insulator has a static dielectric constant, which has a pole at the first critical point $f_{c}=1 / 3$. For a real metal in a composite with a dielectric, the pole is shifted up in the complex frequency plane, turns into a maximum, and eventually becomes negative. We take the real metal dielectric function $\epsilon_{m}$ in the Drude form:

$$
\epsilon_{m}(\omega, f)=1-f \frac{\omega_{p}^{2} \tau(1+i \omega \tau)}{i \omega\left(1+\omega^{2} \tau^{2}\right)} .
$$

Here the plasma frequency $\omega_{p}$ is defined by the electronic concentration of the metal, which is proportional to the metal content $f$. The relaxation rate $\tau^{-1}$ is typically 1 order of magnitude less than the plasma frequency. It may depend, however, strongly on the temperature or the pressure.
Using the EMA equation above for the effective dielectric function of the composite, we find

$$
\epsilon_{\mathrm{eff}}(\omega, f)=\frac{1}{4}\left[\alpha \pm \sqrt{\alpha^{2}+8 \epsilon_{d} \epsilon_{m}(\omega, f)}\right] .
$$

Here the function $\alpha$ has the form

$$
\alpha=(2-3 f) \epsilon_{d}+(3 f-1) \epsilon_{m}(\omega, f) .
$$

This formula is illustrated in Fig. 2, which shows the real part of the dielectric function and the critical line where it becomes negative is clearly visible. The maximum in the region around $f=\frac{1}{3}$ clearly is related to the pole [see Eq. (2)] in the static effective dielectric constant of a composite made out of an ideal metal and a dielectric. For a real metal it turns into a finite maximum as expected.

The superconductor dielectric composites.-The treatment we applied for the intermediate state region is not limited to the metal-insulator composites and MITs. It is easily seen that the nonmagnetic dielectric and superconductor composites show similar behavior. Starting with a small volume fraction of the superconductor $f$, we see that the first superconductive paths appear at the concentration $f_{c}$. The magnetic susceptibility will sense the presence of the superconductor component, but the complete screening of the external magnetic field will occur only at $f \geq f_{d}$, when there is no longer an infinite dielectric cluster present. In the intermediate range of concentrations, the obtained phase is a superconductor and at the same time

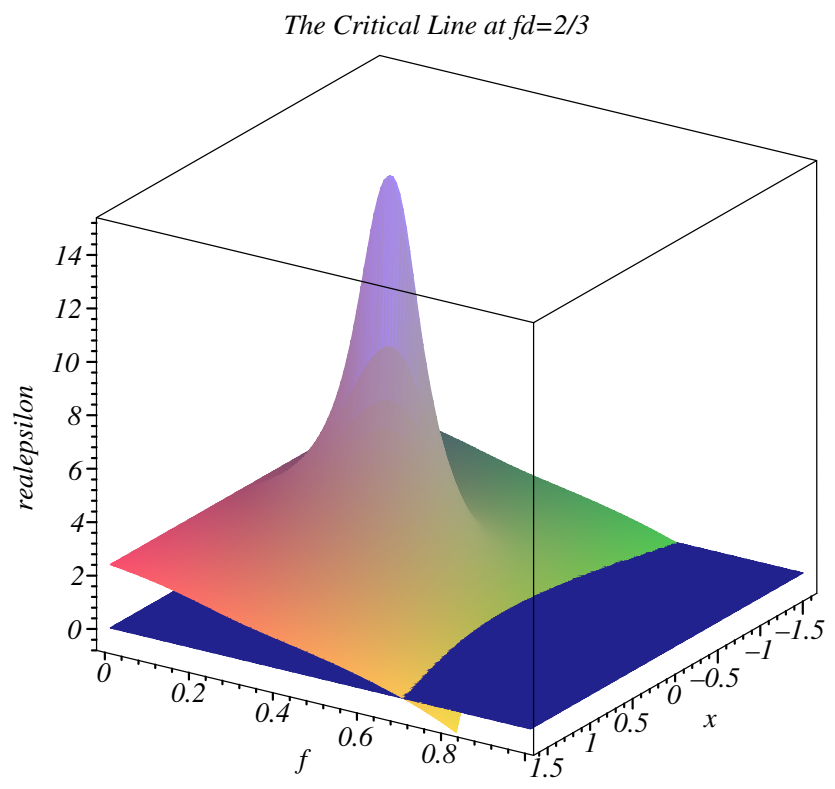

FIG. 2 (color online). The real part of the dielectric function as a function of the metal volume fraction $f$ and the dimensionless frequency $x=\omega \tau$. The critical line at $f_{d}=\frac{2}{3}$ is the cross section of the blue plane defined as $\operatorname{Re} \varepsilon=0$. The dimensionless plasma frequency here is $\omega_{p} \tau=13.7$. The metallic phase is in the region where the real part of the static dielectric function becomes negative-below the horizontal dark plane. 
TABLE II. The dielectric superconductor composites.

\begin{tabular}{ccc}
\hline \hline Dielectric & Phase separation state & Superconductor \\
\hline$\sigma_{\mathrm{dc}}=0$ & $\sigma_{\mathrm{dc}}=\infty$ & $\sigma_{\mathrm{dc}}=\infty$ \\
$P \neq 0$ & $P \neq 0$ & $P=0$ \\
$\chi \leq 0$ & $\chi \leq 0$ & $4 \pi \chi+1=0$ \\
\hline \hline
\end{tabular}

has a nonzero static polarization as a dielectric in the range of temperatures $0 \leq T \leq T_{c}$. This picture is summarized in Table II, where we assume a diamagnetic dielectric. The interphase energy at the two critical points is due to the expulsion of the electric field penetrating the dielectric, but in addition to it now there is present the magnetic field energy density due to the magnetic field screening, which may be partial near $f_{c}$ and complete at $f_{d}$. The penetration depth $\lambda^{-2}$ is proportional to the superfluid density $n_{s}$, and when it is zero the depth is infinite. At low levels of doping the insulating phase of the cuprates plays the role of the dielectric component. At $T=0 \mathrm{~K}$ in the still superconductive samples, the magnetic field penetration depth increases infinitely in the region of small doping concentrations $p$ less than $p=0.1$, the critical concentration $p_{c}$ being close to it. Eventually, the samples are superconductive with a low magnetic field penetration depth equal to infinity. This absence of complete screening of the magnetic field is marked in Table II as negative susceptibility but not vanishing $\mu=1+4 \pi \chi$.

In conclusion, we have shown that in the first order MITs there can be a second critical point, where $\varepsilon_{\text {eff }}=0$. Between the two critical points there is a phase separation state, where two types of extended states are present. In the same way in the superconductor dielectric composites, the dielectric phase and the superconductive one can be also separated by an intermediate phase. The second critical point where the infinite dielectric cluster appears in the metallic phase is observable by means of static polarization studies, by magnetic susceptibility, and by eddy current loss experiments, not to mention different sound velocities and the features in the reflection and/or transmission of electromagnetic waves.
We thank Dr. A. McMahan and the authors of Ref. [12] for kindly giving us permission to reproduce their results in Fig. 1.

*kostadinov.1@osu.edu

[1] P. W. Anderson, Phys. Rev. 109, 1492 (1958).

[2] E. Abrahams, P. W. Anderson, D. C. Licciardello, and T. V. Ramakrishnan, Phys. Rev. Lett. 42, 673 (1979).

[3] Alexander Punnoose and Alexander M. Finkel'stein, Science 310, 289 (2005); Phys. Rev. Lett. 97, 117004 (2006).

[4] P. A. Lee and T. V. Ramakrishnan, Rev. Mod. Phys. 57, 287 (1985).

[5] M. Imada, A. Fujimori, and Y. Tokura, Rev. Mod. Phys. 70, 1039 (1998).

[6] N. F. Mott, Metal-Insulator Transitions (Taylor \& Francis, London, 1990), pp. 17, 156, and 192.

[7] B. L. Altshuler and A. G. Aronov, Modern Problems in Condensed Matter Physics (Elsevier, New York, 1985), pp. $1-151$.

[8] D. Stauffer and A. Aharony, Introduction to Percolation Theory (Taylor \& Francis, London, 1992), p. 13, 2nd ed.

[9] D. J. Bergman and D. Stroud, in Solid State Physics, edited by H. Ehrenreich and D. Turnbull (Academic, Boston, 1992), Vol. 46, p. 147.

[10] T. Kondo, T. Yagi, Y. Syono, Y. Noguchi, T. Atou, T. Kikegawa, and O. Shimomura, J. Appl. Phys. 87, 4153 (2000).

[11] J. R. Patterson, C. M. Aracne, D. D. Jackson, V. Malba, and S. T. Weir, P. A. Baker, and Y. K. Vohra, Phys. Rev. B 69, 220101(R) (2004).

[12] C. S. Yoo, B. Maddox, J.-H. P. Klepeis, V. Iota, W. Evans, A. McMahan, M. Y. Hu, P. Chow, M. Somayazulu, D. Hausermann, R. T. Scalettar, and W. E. Pickett, Phys. Rev. Lett. 94, 115502 (2005).

[13] Yoshimi Mita, Daisuke Izaki, Michihiro Kobayashi, and Shoichi Endo, Phys. Rev. B 71, 100101(R) (2005).

[14] Jan Kunes, Alexey V. Lukoyanov, Vladimir I. Anisimov, Richard T. Scalettar, and Warren E. Pickett, Nature Mater. 7, 198 (2008).

[15] F. G. Shin, W. L. Tsui, and Y. Y. Yeung, J. Mater. Sci. Lett. 8, 1383 (1989).

[16] D. Ishikawa, M. Inui, K. Matsuda, K. Tamura, S. Tsutsui, and A. Q. R. Baron, Phys. Rev. Lett. 93, 097801 (2004). 